\title{
La influencia de la teoría de la dependencia en los discursos de desarrollo de América Latina*
}

\author{
Influence of the Dependence Theory on the Development Discourses in Latin America
}

\author{
Diego Zambrano ${ }^{\text {a }}$ \\ Universidad Internacional de Florida, Estados Unidos \\ dzamb019@fiu.edu \\ ORCID: https://orcid.org/0000-0002-0868-5361
}

DOI: https://doi.org/10.11144/Javeriana.papo25.itdd

Recibido: 04 Septiembre 2018

Aceptado: 03 Abril 2019

Publicado: 29 Junio 2020

\section{Resumen:}

La teoría de la dependencia ha sido considerada como una perspectiva obsoleta para el estudio de la realidad latinoamericana. El éxito de otras áreas geográficas periféricas al posicionarse en el centro de la economía mundial al final del siglo veinte significó el aparente final de la validez de la teoría de la dependencia. Sin embargo, los Estados de América Latina siguen sin ser capaces de corregir las deficiencias estructurales que inspiraron la incepción de la dependencia, ya que continúan siendo significativamente dependientes a la comercialización de materias primas. Este artículo busca reabrir el debate sobre la teoría de la dependencia, con el objetivo de recuperar sus valiosos análisis para la reflexión sobre las tendencias socioeconómicas actuales de la región. A su vez, busca redefinir los aspectos teóricos más rígidos y obsoletos de la teoría de la dependencia, para resaltar su influencia en discursos de desarrollo actuales. En este sentido, este artículo resalta los paralelismos entre la teoría de la dependencia y la literatura académica sobre el desarrollo y los recursos primarios.

Palabras clave: teoría de la dependencia, América Latina, maldición de recursos primarios, comercialización de materias primas, volatilidad de materias primas.

\begin{abstract}
:
Dependence Theory has been deemed as an obsolete approach for the study of Latin-American reality. The success in other peripheral geographic areas that managed to position themselves at the core of the world economy by the end of the 20th century apparently meant that the said theory was not valid anymore. However, the Latin-American countries are still not capable to correct the structural deficiencies that inspired the inception of the dependence, as they are still today significantly dependent on the raw material trading. This paper aims to resume the debate on the Dependence Theory in order to recover some valuable insights for the reflection on the current socioeconomic trends in this region. This work attempts, in turn, to redefine the most rigid and obsolete theoretical aspects of the Dependence Theory in order to underline how it influenced current development discourses. In this vein, this paper highlights the parallel facts between the Dependence Theory and the academic literature on development and primary resources.
\end{abstract}

Keywords: Dependence Theory, Latin America, primary resource curse, raw material trading, raw material volatility.

\section{Introducción}

La relevancia de la teoría de la dependencia ha sido cuestionada en repetidas ocasiones a lo largo de los últimos cincuenta años. Después de haber ganado reconocimiento en los años sesenta y setenta, ha llegado a ser considerada como irrelevante en la actualidad. Packenham (1992) menciona que André Gunder Frank, uno de los autores más reconocidos de la teoría de la dependencia, declaró en 1974 que las ideas de la dependencia, que una vez fueron influyentes, estaban muertas (p. 245). En consecuencia, junto con Gunder Frank, otros académicos como Godfrey (1980) o Sánchez también declararon el fin de la teoría de la dependencia. En este último, se considera que la teoría de la dependencia es apenas mencionada fuera de América Latina, relegada a una nota de pie de página en la literatura académica (Sánchez, 2003, p. 39). El fallecimiento de la teoría

Notas de autor

$$
\text { a Autor de correspondencia. Correo electrónico: dzamb019@fiu.edu }
$$


de la dependencia responde a diferentes tendencias empíricas que ocurrieron en la economía mundial, y a una larga lista de críticas que ha recibido la literatura relacionada al tema. Kay (1989) cataloga muchas de las incontables acusaciones recibidas como:

tautológica, economista, ahistórica, utópica, desprovista de análisis de clase, populista, nacionalista, miope, unidimensional, ideológica, ecléctica, mecánica, sofista, una teleología negativa, idealista, anticapitalista, un estructuralismo marxista, no marxista o no materialista, descuidada en el uso de la teoría marxista, incapaz de romper con el desarrollo burgués, sin bases empíricas, teóricamente imprecisa, poco clara, contradictoria, demasiado global u holística, determinista, metodológicamente y conceptualmente ecléctica - una torre de Babel, carente de claridad, de recomendaciones de política para superar la dependencia, estancamiento, circulación, etc. (p. 175)

Aunque es evidente que la larga lista de críticas recibidas es importante, el fallecimiento de la teoría de la dependencia responde principalmente a varios desarrollos que tomaron lugar en la economía mundial en las últimas décadas. Como lo explica Blaney (1996), el atractivo de la nueva industrialización, la compulsión de nuevas condiciones competitivas, la ejecución hipotecaria de opciones de desarrollo por peonaje de deuda, y el colapso del socialismo disminuyeron la aceptación de la teoría de la dependencia (p. 460). Por ejemplo, los avances industriales de países como Brasil, Argentina, o México presentaron contradicciones empíricas importantes de las ideas de la dependencia ortodoxa de autores como André Gunder Frank. Pero la evidencia más devastadora contra los postulados de la teoría de la dependencia con respecto a la imposibilidad de generar un desarrollo capitalista en países periféricos fue la industrialización de Corea del Sur, Singapur, Taiwán, y Hong Kong. Específicamente, la experiencia de estos países contradice la teoría de la dependencia, ya que esta rechazaba la posibilidad de generar progreso económico en países periféricos a través de su integración avanzada con la economía mundial (Sánchez, 2003, p. 39). Tanto el movimiento de estos países desde su posición periférica al centro de la economía mundial, como la relativa industrialización de varios países latinoamericanos supusieron las mayores razones en contra de la relevancia de la teoría de la dependencia.

La popularización de los paradigmas que sucedieron a la dependencia y su posición actual dentro de la literatura académica invitan a pensar que la realidad de América Latina ha validado el rechazo de los postulados de la teoría. Sin embargo, la región continúa manteniendo una constante dependencia a la comercialización de materias prima. Es más, desde la supuesta muerte de la teoría, la región "ha profundizado la dependencia externa de la mayoría de los países y se ha ensanchado el hiato que los separaba de las metrópolis" (Beigel, 2006, p. 308). Este contexto moderno invita a reabrir el debate de la dependencia como un marco explicativo relevante para la realidad actual de la región. Se considera empíricamente incorrecto determinar a la teoría de la dependencia como muerta o irrelevante dadas las condiciones del modelo económico actual latinoamericano. Es por este motivo que este artículo decide articular un nuevo entendimiento de la teoría con base en una revisión de sus debates principales, una redefinición de su alcance teórico, y una examinación de su influencia en discursos actuales sobre los problemas económicos de la región. Para lograr dicho objetivo, este artículo se divide en cuatro secciones: la siguiente presenta una descripción breve de las tendencias actuales en materia de política económica de América Latina como justificación para reabrir el debate de la dependencia. La segunda sección presenta una discusión de los principales debates de la teoría de la dependencia, y avanza en su concepto y marco teórico para adelantar su aplicabilidad a la realidad actual. Luego, se resalta la influencia de la teoría de la dependencia en la literatura académica sobre el rol de las materias primas en el desarrollo. En este sentido, las siguientes secciones introducen conceptos específicos de la literatura de las materias primas dentro de un marco teórico de dependencia, acentuando la influencia y la relevancia de la misma en el estudio de la realidad latinoamericana. Como conclusión, el artículo reflexiona sobre la posibilidad de pensar en un paradigma de dependencia que trasciende enunciados teóricos de causalidad, y se presenta como un movimiento o acercamiento para el estudio de la economía mundial desde la perspectiva de la periferia. 


\section{El contexto moderno latinoamericano y la teoría de la dependencia}

En la actualidad, cualquier discusión sobre la dependencia procede principalmente como una lectura del acta de defunción de la teoría (Blaney, 1996, p. 460). Sin embargo, las tendencias de los procesos socioeconómicos de América Latina invitan a revisitar la validez de sus postulados. La paradoja de los conceptos teóricos de la dependencia es que "mientras las categorías cayeron vertiginosamente en desuso, las realidades del imperialismo han sido más vívidas e impresionantes" (Beigel, 2006, p. 360). Una revisión de la política económica de la región, desde la caída de la teoría como un marco teórico para el entendimiento de la realidad latinoamericana, demuestra la relevancia de la dependencia. En primer lugar, la crisis de la década de los años ochenta, conocida como "la década perdida" de Latinoamérica, debido a sus devastadoras consecuencias, sucede en un contexto de desaceleración económica de la comercialización de materias primas. Las reformas neoliberales de los años noventa lograron controlar los desajustes macroeconómicos de la región, pero incrementaron los índices de desigualdad y pobreza que desataron diferentes crisis políticas en Argentina, Bolivia, o Venezuela. Este contexto social, político, y económico pavimentó la llegada de varios movimientos políticos progresistas a lo largo de la región, que por diversas razones mejoraron los índices de pobreza y desigualdad. Por ejemplo, los niveles de pobreza en América Latina disminuyeron de un estimado de doscientos veintiún millones de personas en el año 2002, a ciento setenta y cinco millones en el año 2017 (OECD et al., 2016; Smith, 2012, pp. 228-229). Similarmente, entre los años 2000 y 2012, la desigualdad de ingresos disminuyó en un promedio de tres puntos en el coeficiente GINI (Tsounta y Osueke, 2014, p. 7). Sin embargo, las tendencias socioeconómicas de los últimos años, con incrementos en los índices de pobreza y desigualdad, son preocupantes e invitan a revisitar la validez de argumentos de la dependencia que han sido considerados irrelevantes.

De acuerdo con el reporte del desarrollo humano de América Latina y el Caribe del Programa de las Naciones Unidas para el Desarrollo (2016), casi treinta millones de las personas que mejoraron sus condiciones socioeconómicas entre el año 2000 y el año 2015 caerían en la pobreza de nuevo. A su vez, la región presentó una contracción económica tanto en el año 2015 como el año 2016, una tendencia que no sucedía desde inicios de los años ochenta. Esta contracción puso a doscientos millones de personas en la región en riesgo de caer en condición de pobreza, posibilidad preocupante, ya que el $64 \%$ de los jóvenes en América Latina vienen de hogares pobres o económicamente vulnerables (Tavares, 2017). Aunque la magnitud de estas circunstancias socioeconómicas no se compara con la de las dificultades de "la década perdida", la desaceleración económica actual también responde a una contracción en la comercialización de las materias primas. Por ende, es necesario evaluar la validez de los desarrollos empíricos que aparentemente condenaron la validez de la teoría de la dependencia, dado que el momento actual de la región presenta similitudes con otras instancias de problemas socioeconómicos como consecuencia de la dependencia a la comercialización de materias primas.

Por ende, es importante discutir la validez de las razones detrás del fallecimiento de la teoría de la dependencia. En primer lugar, Evans (1987) problematiza el uso de la experiencia de los países del este de Asia como excusa para atacar la validez de la teoría de la dependencia. Esto es por la necesidad de reconocer el rol del contexto en el cual dichas economías lograron moverse fuera de la periferia, principalmente debido al soporte de los países del centro, que no ha sido extendido a América Latina. A la vez, es importante resaltar que aquellos que utilizaron la experiencia asiática como contrapeso a los postulados de la dependencia, no ajustaron la validez de sus observaciones una vez esta región sufrió una crisis financiera en los años noventa. Interpretaciones contemporáneas sobre el llamado "milagro económico" de las economías del Sudeste Asiático contradicen el rechazo de la dependencia. Por el contrario, estas invitan a un refinamiento de la teoría, al enfocarse en las interacciones entre la dependencia externa y las instituciones locales (Bull y Bøäs, 2012, p. 324). En segundo lugar, la relativa industrialización de Argentina, Brasil, y México en los años setenta, que representó una contradicción empírica a los postulados de la dependencia, ya no es 
relevante. Por el contrario, la tendencia moderna de la región es la profundización del modelo extractivo, que ha incrementado el rol de las materias primas en la actividad económica de la región. Por ejemplo, países como México, Argentina, Brasil, Ecuador, Perú, Venezuela, Colombia, Uruguay, y Bolivia han incrementado la proporción de productos primarios en las exportaciones totales entre el año 2000 y el año 2011 (Burchardt y Dietz, 2014, pp. 472-473). Esta dinámica también ha ocurrido con la proporción del sector primario en el producto interno bruto de la región con la excepción de Colombia y Ecuador. Los movimientos hacia la desindustrialización en América Latina ya fueron observados por Hirschman (1987) desde principios de los años setenta en Chile Argentina, y México (pp. 13-19). En consecuencia, se observa una drástica desindustrialización en la región que, en vez de contradecir los postulados de la dependencia, invita a revisitar el movimiento.

Sin embargo, la razón más importante para reabrir el debate sobre la teoría de la dependencia yace en las tendencias socioeconómicas actuales de la región. Aunque es ingenuo negar las mejoras materiales de los latinoamericanos en este siglo veintiuno, es indispensable identificar las razones detrás de dichas mejoras. Las tendencias socioeconómicas modernas de América Latina son consecuencia del crecimiento económico de China, que ha sido alimentado por un consumo masivo de materias primas, lo que ha aumentado la demanda de estos productos en el mercado internacional y, como consecuencia, su precio. Esta dinámica ha favorecido de manera importante a los países de América Latina, ya que la región concentra su actividad económica exportadora en la extracción y comercialización de materias primas a China (Smith, 2012, pp. 228-229). Cuando la expansión económica de China desaceleró, los precios de las materias primas y su subsecuente consumo disminuyó dramáticamente, lo que afectó las economías de la región. Los procesos de intercambios económicos que han surgido entre China y América Latina en la última década se asemejan a una relación de dependencia, como fue descrita por los autores principales de la teoría. La industrialización agresiva de China ha transformado las relaciones de dependencia con la región, creando nuevas condiciones de dependencia y reduciendo los incentivos estructurales para la industrialización latinoamericana (Bull y Bøás, 2012, p. 332). Siguiendo el argumento de Ortiz (2012), la sostenibilidad económica a largo plazo de la región está en riesgo debido al incremento de su dependencia a las exportaciones de materias primas a China (p. 188). De hecho, si esta dependencia continua a los mismos niveles de los años entre 1984 y 2008, esto significa que para que la región crezca económicamente en un $1 \%$ anual, las exportaciones de materia prima a China deberían crecer cerca de un 0,12\% al año (Ortiz, 2012, p. 187). A simple vista, la relación económica de China y América Latina se asemeja al "desarrollo dependiente" descrito por autores como Dos Santos (1970) o Cardoso y Faletto (1979). Esta realidad latinoamericana contemporánea presenta el contexto idóneo para reabrir el debate de la dependencia, justificando al menos una nueva discusión sobre sus postulados teóricos.

\section{Sobre la dependencia}

Es difícil determinar una definición general y totalizante sobre la teoría de la dependencia. En términos generales, se puede asumir que se refiere a un contexto analítico dentro de la tradición marxista, con el cual se estudia la expansión del sistema capitalista mundial y sus implicaciones desde el punto de vista de los países periféricos. Como su observación principal, la dependencia denuncia las asimetrías de las relaciones capitalistas entre el centro y la periferia, y enumera las dificultades para el desarrollo a las que se enfrentan los países de América Latina, dada su localización dentro de las cadenas de producción global. Por ende, el nacimiento de la dependencia como un paradigma analítico dominante en los años sesenta y setenta representó una crítica importante a la teoría de la modernización y, a su vez, un avance en el estudio del imperialismo (Packenham, 1992, p. 18). Sin embargo, la dificultad para conceptualizar a la dependencia, en términos generales, nace de los importantes debates que existieron entre autores que se identificaron dentro de los postulados de la teoría. Por ejemplo, dentro del movimiento existían autores que compartían el diagnóstico 
sobre la condición periférica de la región, enunciados por Raúl Prebisch y la Comisión Económica para América Latina y el Caribe (Cepal), pero presentaban grandes contradicciones con las prescripciones políticas de los mismos (Packenham, 1992, p. 17). A su vez, existen importantes debates conceptuales y teóricos entre autores como Serra, Cardoso, y Rui Marini, quienes debatieron importantes ideas sobre la dependencia como el intercambio desigual, la explotación del trabajo, o el subimperialismo (Kay, 1989, pp. 164-170). Sin embargo, la división más importante dentro de la teoría de la dependencia existe entre lo que Packenham (1992) define como dependencia ortodoxa y dependencia no ortodoxa (pp. 31-32). Los mayores exponentes de dichas calificaciones son André Gunder Frank y Fernando Cardoso, respectivamente. Su debate se centra en la teoría del subdesarrollo enunciada por Frank (1967), quien dictamina que el desarrollo capitalista es imposible en la periferia, y que el subdesarrollo periférico es un resultado inevitable del desarrollo del centro, condenando a la periferia al subdesarrollo (p. 11). Cardoso critica esta postura, indicando que mientras la expansión del capitalismo en la periferia es desigual, este no es imposible. Por eso, Cardoso (1973) avanza en la idea del desarrollo dependiente como un concepto analítico para explicar la expansión capitalista en los países del tercer mundo (p. 143). Siguiendo con la idea de Cardoso, Palma (1978) critica la postura de Frank, y argumenta que la dependencia no es la causa del subdesarrollo, sino una condición que profundiza los problemas del subdesarrollo (p. 903). Este debate es, tal vez, el más importante de la dependencia, ya que la inmensa mayoría de las críticas contra la teoría dentro del mundo de habla inglesa ha sido contra el trabajo de Frank. Pero la división cobra aún más importancia ya que las críticas al trabajo de Frank se han asumido como de aplicación a toda la teoría de la dependencia, sin explorar los debates y las importantes diferencias que existen entre los autores (Kay, 1989, p. 174). Esta práctica de generalización por parte de los críticos, sumada a los incontables debates entre autores, ha contribuido a la irrelevancia y a la imposibilidad de describir a la dependencia en términos generales.

Tal vez, el mayor problema de la teoría de la dependencia radica en su nombre y en las expectativas que genera. La denominación de un variado grupo de trabajos presentando un marco analítico para estudiar el capitalismo en el tercer mundo como teoría es sencillamente problemático. Una teoría implica un enunciado hipotético de causa y efecto, la expectativa de que, ante la presencia de un factor causal, el académico ha de observar un efecto o resultado. Tanto teórica como metodológicamente, los autores de la dependencia rechazan esta postura, y escriben, en cambio, sobre una realidad o una condición en la que el capitalismo se desempeña. Esta aclaratoria no busca disminuir o menospreciar las válidas críticas que se han elevado en contra de muchos de los argumentos dentro de la teoría de la dependencia, como los resumidos por Kay (1989, pp. 174-196). Sin embargo, lo que busca es precisar el entendimiento de un rico cuerpo de trabajo académico, y rescatar su validez como un marco teórico con el cual entender la realidad latinoamericana actual. Para ello, es importante resaltar que la teoría de la dependencia no es una teoría, es un movimiento o acercamiento académico que busca estudiar y entender el capitalismo, basada en una serie de principios y presunciones básicas y compartidas. Por ende, antes de intentar expandir en una conceptualización, es útil identificar los aspectos de continuidad presentes entre los autores, que permiten enmarcar a la dependencia como un movimiento que trasciende una teoría.

El primer aspecto de continuidad o presunción que mantiene una coherencia en el movimiento de la dependencia es la idea de la división del trabajo internacional. Los autores de la dependencia comparten la visión de la división del trabajo en el mercado internacional como un proceso condicionado por la expansión del capitalismo y, por ende, es un proceso condicionado por la dependencia de la periferia (Jaguaribe et al., 1968, p. 180). Contrario al dogma capitalista, el movimiento de la dependencia considera que la división del trabajo internacional no generó un desarrollo o progreso paralelo entre las áreas que se especializaron en diferentes procesos productivos (Muñoz, 1981, p. 26). La consecuencia de la división del trabajo internacional fue una diferencia en el nivel de desarrollo de los distintos sectores geográficos, que significó una ventaja o mayor beneficio a aquellos que se especializan en procesos productivos de valor agregado. El segundo elemento de continuidad en el movimiento de dependencia es la idea de la estructura internacional, que 
afecta las relaciones internacionales de las distintas áreas geográficas y entidades políticas. En este sentido, Muñoz (1981) argumenta que la posición de una entidad política dentro de la estructura internacional condiciona su desarrollo (p. 25). Por otro lado, autores dentro del movimiento se enfocan en el estudio de la influencia externa y el desarrollo de las entidades políticas de acuerdo con su posición en la estructura internacional. Caporaso y Zare (1981) argumentan que el objeto de estudio de la dependencia es explorar las asimetrías en las relaciones internacionales, y cómo su posición en la estructura internacional condiciona o beneficia su capacidad de influencia (p. 44). Más allá de estas continuidades de contenido, Packenham (1992) también identifica una presunción compartida de rechazo contra teorías, conceptos, o marcos analíticos que emergieron de la experiencia del mundo industrializado (pp. 29-30). El movimiento de la dependencia tiene una posición metodológica y epistemológica contraria a la teoría de la modernización. La dependencia considera a la modernización como el resultado del estudio de la realidad del mundo industrializado, haciéndolo irrelevante para el contexto de los países del tercer mundo. Por ende, el movimiento de la dependencia representa una iniciativa para construir un marco teórico y analítico que emerge de la realidad empírica de la periferia, y que es capaz de entenderla y cambiarla. Finalmente, el movimiento de la dependencia presenta una continuidad en la premisa filosófica de entender el trabajo como la actividad fundamental de la humanidad, haciéndola el eje del estudio del comportamiento humano (Palma, 1978, p .883). Es este sentido, Packenham (1992) identifica al movimiento de la dependencia dentro del Marxismo, dándole una coherencia como movimiento de estudio del capitalismo (p. 28).

Una vez delimitadas algunas de las más importantes continuidades y presunciones del movimiento de la dependencia, es consecuente establecer un marco conceptual que permita capturar un objeto de estudio central, sin eliminar la diversidad y el debate que ya existe entre diferentes autores. Por ende, si entendemos a la dependencia como un movimiento en vez de una teoría, en el que gravitan diferentes autores estudiando diferentes aspectos de la expansión del capitalismo en la periferia, partiendo de un núcleo de principios fundamentales, entonces es necesario establecer una idea general sobre lo que implica hablar de dependencia. Si bien ya se delimitó que la dependencia no es una teoría, en cuanto que no presenta una relación causal observable; y, si bien ya se estableció que el movimiento de la dependencia asume el estudio del trabajo como actividad fundamental humana y examina cómo su división afecta e influencia las relaciones entre entidades políticas, entonces el siguiente paso lógico es darle un cuerpo ontológico a la dependencia. Para esto es útil revisitar las definiciones presentes en los escritos de algunos autores que se identifican con la dependencia. De acuerdo con Heraldo Muñoz (1981), la dependencia es un acercamiento con el que se estudia cómo las relaciones de dependencia dentro de la estructura capitalista mundial influencian el desarrollo de las naciones (p. 45). En consecuencia, esta es entendida como una condición por la cual fuerzas estructurales externas afectan los procesos internos de una entidad política, como lo es el desarrollo. Para Raymond Duvall (1978), la dependencia se refiere a una situación en la cual el desarrollo es afectado por diferentes procesos del contexto capitalista (pp. 58-59). Duvall considera que la dependencia es una condición general dentro de la cual ciertas relaciones toman lugar, en vez de ser un concepto contenedor de datos o una causa explicativa (p. 52). Por consiguiente, entender a la dependencia como un movimiento en vez de una teoría implica definirla como una situación condicionante, un contexto estructural que afecta los procesos y las relaciones de ciertos entes políticos dentro de la estructura internacional.

Ahora bien, si la dependencia es una situación condicionante, entonces ¿cuáles son los aspectos que esta condiciona y cuáles son las razones que generan dicho condicionamiento? Con respecto al objeto del condicionamiento de la dependencia, los autores del movimiento identifican al desarrollo de los países periféricos como el proceso que es afectado por dicha situación condicionante. Para Theotonio dos Santos (1970), la situación condicionante de dependencia es un entorno en el que el desarrollo de un economía está condicionada por el desarrollo y la expansión de otra (p. 231). Sin embargo, lo que diferencia a la situación de dependencia de una interdependencia es la asimetría de dicha relación. En este sentido, una situación condicionante de dependencia existe cuando hay una relación asimétrica, en la que una economía presenta una 
expansión autónoma, mientras que la otra presenta un reflejo, ya sea positivo o negativo, de la autonomía de la economía dominante. El factor determinante de dicha asimetría es el proceso de expansión del capitalismo, ya que este determina la división del trabajo en la economía mundial, y por ende esculpe la direccionalidad de la influencia de la situación condicionante de dependencia. Como consecuencia, esta definición de dependencia implica la posibilidad de cambio, ya que el proceso de la expansión del capitalismo es continuo, y moldea las especificidades de la situación condicionante de dependencia y su direccionalidad (Muñoz, 1981, p. 27; Jaguaribe et al., 1968, p. 182). El objeto de estudio del movimiento de dependencia es el proceso de expansión capitalista, cómo este delimita la situación condicionante de dependencia y su direccionalidad de acuerdo con las asimetrías económicas entre entidades políticas debido a la configuración de la división del trabajo en la economía global. Finalmente, el movimiento de la dependencia se refiere a las interacciones y los procesos dentro del contexto de dependencia y cómo estos condicionan el desarrollo de la periferia.

Una vez dicho esto, la dependencia como un marco analítico para entender la realidad empírica actual cobra una relevancia que emerge de la necesidad de articular procesos e interacciones actuales dentro de un marco teórico capaz de capturar las dinámicas descritas anteriormente. En este sentido, las tendencias socioeconómicas de desaceleración y disminución presentes en América Latina, como consecuencia de los cambios en la economía China, se entienden dentro de un contexto analítico de dependencia. Dichas dinámicas presentan paralelismos con los procesos de desarrollo económico forzado y distorsionado descritos por Duvall (1978, p. 70). Los cambios políticos que caracterizaron a la región en los años noventa, a principios del siglo veintiuno, y los que están ocurriendo actualmente pueden ser explicados dentro de los parámetros mencionados por muchos autores de la dependencia, como resultado de distorsiones sociopolíticas (Duvall, 1978, p. 70). Sin embargo, la relevancia del movimiento de la dependencia radica mayoritariamente en su capacidad para contextualizar, una vez que la ha influenciado, a la creciente literatura sobre las economías de enclave y a los problemas de las economías concentradas en la explotación de materias primas. Estos aspectos son explorados a fondo en las siguientes secciones.

\section{La influencia de la dependencia y su relevancia actual}

Como fue expuesto anteriormente, la realidad de la política económica latinoamericana actual se caracteriza por la explotación y comercialización de materias primas. Si bien esto ha sido una característica histórica de la región, es importante reiterar la centralidad de dicha actividad en la actividad comercial de la región en el siglo veintiuno. En estos últimos quince años, la región ha incrementado su actividad de explotación y comercialización de materias primas en términos de sus exportaciones y de su economía general, como consecuencia de la expansión del capitalismo. La región ocupa una posición específica en la división de trabajo internacional que caracteriza su actividad económica general y, más específicamente, su desarrollo. Dicha posición en la división de trabajo influyó en el tipo de relación comercial que Latinoamérica entablo con China, una vez este país emprendió una transformación de su posicionamiento en la economía mundial. Esta dinámica ya ha sido explicada anteriormente, así que lo importante ahora es enmarcar esta relación dentro de una situación condicionante de dependencia. En primer lugar, la industrialización de China le permitió moverse de la periferia de la economía mundial al centro. Este movimiento, junto con la naturaleza capitalista de la estructura económica internacional en la que ocurrió, condicionaron los procesos de comercialización de las materias primas con América Latina. Este proceso ocurrió a través de tres vías: 1) el crecimiento económico de China incrementó la demanda de productos primarios, lo que aumentó su precio y su producción; 2) América Latina se concentró en la explotación y exportación de estas materias primas para satisfacer la demanda generada por el crecimiento económico China; 3) la relación de este intercambio comercial entre China y América Latina se caracterizó por la subordinación periférica y la vulnerabilidad por los cambios en la economía dominante. América Latina presentó sensibilidad y vulnerabilidad a los cambios de la economía 
China, por lo que condicionó su desarrollo y limitó su sostenibilidad, ya que la relación de intercambio fue asimétrica debido a sus respectivas posiciones en el mercado global. La vulnerabilidad y la sensibilidad de las relaciones entre dos países no son necesariamente dependientes (Duvall, 1978, pp. 62-65), pero es indispensable identificar si estas responden a subordinación o compromiso por parte de la periferia (Dos Santos, 1973, pp. 78-79).

Si bien es cierto que la dinámica actual de América Latina invita a reabrir el debate de la dependencia, también es importante examinar los procesos por los cuales la comercialización de las materias primas condiciona al desarrollo, y como este condicionamiento existe en un contexto de dependencia. Para ello, es útil examinar la literatura sobre la comercialización de las materias primas y cómo esta afecta al desarrollo. Esta revisión muestra, no solo cómo las estructuras y las relaciones modernas condicionan el desarrollo de países periféricos, sino también cómo estos procesos y sus respectivos estudios muestran paralelismos importantes con los postulados de la dependencia, resaltando su influencia y relevancia. La literatura sobre la comercialización de materias primas es amplia, con notables ejemplos en los trabajos de Corden (1984), Corden y Neary (1982), Sachs y Warner (1995), Deaton (1999), Blattman et al. (2007), y Bahar y Santos (2018). Estos trabajos identifican los mecanismos principales por los cuales la comercialización de las materias primas genera desbalances económicos en los países que se enfocan en la explotación de estos recursos y condicionan su desarrollo. En primer lugar, la comercialización de las materias primas afecta a los países que se concentran principalmente en su explotación a través de la apreciación del tipo de cambio. La comercialización de las materias primas incrementa la demanda por la moneda local, lo que hace que su valor suba con relación a la moneda de intercambio en el mercado global (Corden, 1984; Corden y Neary, 1982). La apreciación de la moneda local hace que las exportaciones de todos los sectores sean más costosas, lo que lleva a que la actividad exportadora se contraiga. A su vez, la sobreapreciación de la moneda local gracias a la comercialización de las materias primas hace que las importaciones sean más económicas, y por consecuencia más atractivas. Esto genera incentivos para que el mercado interno se enfoque en importar productos en vez de apoyar la producción nacional. La estructura de incentivos generada por la comercialización de las materias primas genera una pérdida de conocimiento productivo en sectores que no son de extracción, limitando la productividad real de la economía local y condicionando su desarrollo a través de ciclos inestables (Sachs y Warner, 1995). Entre estos incentivos se encuentra la necesidad de mantener el enfoque económico en la comercialización de las materias primas para obtener ganancias en moneda extranjera, y así equilibrar los déficits comerciales generados por las asimetrías de los términos de intercambio.

Como ya se comentó anteriormente, los países de América Latina están enfocados en la comercialización de materias primas como producto de la expansión del capitalismo. Cuando este se extendió hacia América Latina a través del colonialismo, las economías locales fueron estructuradas, principalmente para abastecer las necesidades de la economía colonial (Gordon, 1965, p. 12). Este factor condicionó la forma en la que región se insertó a la economía mundial luego de la revolución industrial, ya que América Latina se concentró en proveer la materia prima necesaria para la industrialización de Europa (Furtado, 1973, p. 38). La región ha sido incapaz de abandonar su posición como exportador de materias primas, manteniendo un contexto de relaciones asimétricas a través de los mecanismos condicionantes de la comercialización de materias primas. Los mecanismos descritos anteriormente, y que abundan en la literatura sobre países exportadores de materias primas, muestran paralelismos importantes con el movimiento de la dependencia. Tanto la sobreapreciación de la moneda local y sus respectivos desbalances económicos, como la pérdida de productividad económica, producto de la sobreconcentración en la explotación, son consecuencias de la posición de la región como proveedor de materias primas en la división de trabajo internacional. Muchos otros países en el mundo tienen presencia de explotación de recursos primarios, pero estos logran mitigar sus efectos gracias a su posición dentro de la división del trabajo en la economía mundial. Pero para países que concentran la mayoría de su actividad económica en la exportación de materias primas, estos mecanismos condicionan su desarrollo, ya que limitan sus opciones de políticas públicas y los hacen sensibles a cambios en el mercado. 
La vulnerabilidad de los países en situación condicionante de dependencia a cambios en el mercado global es el segundo mecanismo por los cuales la comercialización de las materias primas genera desbalances económicos en los países que se enfocan en la explotación de estos recursos y condicionan su desarrollo. La literatura sobre la comercialización de las materias primas identifica la volatilidad de los precios en el mercado internacional como un mecanismo condicionante para el desarrollo de estos países. Autores como Blattman et al. (2007), Deaton (1999), y van der Ploeg y Poelhekke (2009) argumentan que una alta concentración de actividad económica en la exportación de recursos primarios expone a estos países a un gran riesgo financiero, ya que los precios de las materias primas tienden a fluctuar dramáticamente en el mercado internacional, lo que condiciona su desarrollo, ya que limita su capacidad de planificación y estabilidad en momentos de cambios drásticos. Siguiendo esta línea, Goderis y Malone (2011) ahondan en esta discusión, ya que presentan un modelo estadístico capaz de predecir desbalances económicos a través de la fluctuación y la especulación de los precios de las materias primas. Estos desbalances condicionan el desarrollo de los países exportadores de materias primas, ya que estos presentan ciclos de crecimiento económico durante instancias de altos precios que desaparecen a largo plazo producto, debido a fluctuaciones y especulaciones sobre los precios.

Este mecanismo de condicionamiento del desarrollo de estos países presenta importantes paralelismos con el movimiento de la dependencia y con la realidad empírica de América Latina. Como ya se mencionó anteriormente, la razón detrás del condicionamiento de la región a la exportación de recursos primarios yace en la expansión del capitalismo y su posición dentro del mercado global. Sin embargo, el factor determinante del condicionamiento del desarrollo de países periféricos debido a la comercialización de recursos primarios es la vulnerabilidad a la volatilidad de los precios. Este mecanismo se encuentra en dos instancias de la realidad empírica de América Latina, ya mencionadas en este artículo. La primera es "la década perdida", que se refiere a la crisis socioeconómica desatada en la región, producto de una crisis de deuda pública generada por la fluctuación dramática de los precios del petróleo. La segunda es el momento actual en el que la región ha visto cómo los avances socioeconómicos de la primera década del siglo veintiuno se han ido disminuyendo debido a fluctuaciones drásticas en los precios de las materias primas, como consecuencia de la desaceleración económica de China. Toda esta dinámica es producto de los postulados de la dependencia, ya que América Latina no sería vulnerable a estas fluctuaciones si tuviese otra posición en la división del trabajo en la economía mundial; la región no tendría esa posición si la expansión capitalista hubiese tomado otro proceso que no fuese la colonización y la subsecuente revolución industrial; y la comercialización de las materias primas no generaría condicionamientos al desarrollo de la periferia si no estuviese sujeta a las dinámicas de mercado del capitalismo internacional. Por ende, es evidente que la dependencia sigue teniendo relevancia en la explicación de procesos y relaciones modernas, y que esta ha influenciado y mantiene paralelismos con importantes discursos actuales sobre desarrollo.

No obstante, la influencia y la relevancia del movimiento de la dependencia es aún más evidente al examinar la literatura de la maldición de los recursos, o el "resource curse", por su denominación en inglés. Esta literatura discute cómo la comercialización de materias primas condiciona el desarrollo de los países, particularmente los mecanismos que distorsionan los procesos políticos e institucionales, con notables ejemplos como Bourguignon y Verdier (2000), Acemoglu et al. (2004), Isham et al. (2005), y Boschini et al. (2007). El primer mecanismo por el cual la comercialización de las materias primas condiciona el desarrollo de los países es la limitación de adaptar políticas redistributivas que beneficien a las clases medias, restringiendo la adopción de políticas de crecimiento (Bourguignon y Verdier, 2000). Esta lógica identifica los incentivos políticos que genera la explotación de materias primas como principal actividad productiva de un país, ya que esto genera los mayores beneficios para aquella minoría que controla dichas actividades, y más aún si estas son reguladas por el estado. De hecho, tanto Isham et al. (2005) como Acemoglu et al. (2004) argumentan que la presencia de importantes recursos naturales explotables genera incentivos para que las élites controlen el poder a través de la destrucción de instituciones políticas, mediante la pacificación de la disidencia por medio del soborno, la evasión de contraloría pública como consecuencia de las débiles instituciones, y la acción de 
evitar la modernización y el desarrollo, perpetuando estructuras internas de explotación. En este sentido, Boschini et al. (2007) discuten cómo la naturaleza del recurso abundante afecta los mecanismos de distorsión política mencionados anteriormente. La relativa facilidad o dificultad para explotar un recurso y apropiarse de sus beneficios comerciales condiciona el comportamiento de las élites y su control sobre el Estado. Si el recurso es de fácil acceso, y la apropiación de su beneficio comercial es posible, entonces las élites incurrirán en comportamientos destructivos para el desarrollo sostenible y duradero, como la búsqueda de rentas en detrimento de otras políticas productivas, y el soborno para lograr su accionar, eliminando la competencia política. Estos procesos políticos e institucionales logran condicionar el desarrollo de dichos países, ya que mantienen las estructuras internas que lo afectan.

Los retos institucionales y políticos que presenta la comercialización de las materias primas, discutidos por la literatura de la maldición de los recursos, han sido ampliamente debatidos por autores dentro del movimiento de la dependencia. En primer lugar, es interesante discernir sobre la implicación teórica de los mecanismos descritos anteriormente. El análisis de cómo explotar y exportar materias primas influencia el comportamiento político de actores a nivel interno es el principal objeto de estudio del movimiento de la dependencia. En la sección anterior se discute cómo una de las continuidades y presunciones más importantes del movimiento es el consenso para estudiar cómo las estructuras externas influyen y condicionan el desarrollo de las estructuras internas de los países periféricos. Esto es exactamente lo que hace la literatura de la maldición de los recursos, cuando atribuye el comportamiento interno de las elites políticas y del desarrollo institucional a los incentivos que existen para comercializar recursos en las estructuras externas. Es más, las dinámicas descritas dentro del marco analítico de la literatura del "resource curse" pueden ser vistas como una expresión del trabajo de Cardoso y Faletto (1979) sobre la dependencia interna y la dependencia externa. Estos autores explican cómo los procesos y las estructuras de dependencia interna mantienen a América Latina en una posición de dependencia externa, y cómo esa posición influye y condiciona el accionar de las estructuras domésticas. Muchos de los postulados de esta literatura ya fueron ampliamente estudiados por el movimiento de la dependencia. Autores como Palma (1978) reflexionan sobre los procesos que limitaron el desarrollo del capitalismo en América Latina, indicando que una vez la región trascendió el dominio colonial, las élites locales mantuvieron la situación condicionante de dependencia a través de su alineación con los intereses externos y la imposición de estructuras que distorsionan el desarrollo, pero mantienen su beneficio y la situación de dependencia gracias al control del Estado (pp. 896-897). Por consiguiente, la lectura de discursos de desarrollo actuales, como la literatura de la maldición de los recursos, muestra la influencia y la relevancia del movimiento de la dependencia en el estudio y la explicación de la realidad de los países periféricos.

\section{Conclusión}

La realidad de la política económica actual de América Latina presenta rasgos similares a aquellas que inspiraron el inicio de la teoría de la dependencia. La región continúa teniendo una posición de proveedor de materias primas dentro de la división del trabajo internacional. A su vez, los países latinoamericanos continúan presentando importantes retos socioeconómicos como la pobreza o la desigualdad. Importantes autores señalan a la posición de la región dentro de la división del trabajo internacional como la causa de sus distorsiones socioeconómicas (Bulmer-Thomas, 1994, p. 15-17; Skidmore y Smith, 1992). Sin embargo, la dinámica más clara de la relevancia de la dependencia en el estudio actual es el movimiento de China dentro de la economía mundial. En primer lugar, la experiencia China presenta importantes evidencias empíricas en contra del determinismo de algunos postulados de la dependencia. Es por esta razón que, como ya expusieron autores como Cardoso, Faletto, Duvall, o Furtado, entre muchos otros, la relevancia de la dependencia pasa por el abandono del determinismo sobre el desarrollo de la periferia. Además, la relación comercial que China ha entablado con América Latina también resalta la importancia de las ideas de la dependencia. 
Es importante contrastar cómo, si asumimos a la dependencia como una situación condicionante para el desarrollo, el aceleramiento y la desaceleración del consumo de materias primas por parte de China condiciona la direccionalidad, magnitud, y sostenibilidad del desarrollo de la región. Por esto, la reevaluación de la validez de los postulados de la dependencia se vuelve relevante.

Sin embargo, la revisión de distintas narrativas sobre desarrollo, que estudian el rol de los recursos naturales en países del tercer mundo, muestra no solo la relevancia de los postulados de la dependencia, sino también su validez e influencia. La literatura sobre las distorsiones económicas generadas por la comercialización de las materias primas sigue la lógica de muchas ideas debatidas por los autores de la dependencia. Sin embargo, la mayor demostración de la validez, influencia, y relevancia de los postulados de la dependencia se observa en la literatura sobre la maldición de los recursos, que describe los mecanismos mediante los que la comercialización de las materias primas distorsiona el desarrollo político e institucional de los países, y cómo esto termina condicionando su desarrollo económico. Esta dinámica fue discutida por los mayores exponentes de la dependencia para explicar la problemática de la política económica de América Latina. Muchos autores dentro de la literatura del "resource curse" incluso invitan a la estabilización macroeconómica y a la diversificación de la producción exportadora, donde estas prescripciones inspiraron la dependencia y fueron enunciadas dentro de la misma. Lo que resulta aún más relevante es que importantes sectores que estudian el desarrollo actualmente lo hacen desde un enfoque global, analizando cómo la configuración del sistema internacional afecta a los países del tercer mundo. El estudio de cómo las estructuras globales afectan, influyen, o condicionan el desarrollo de los países del tercer mundo es, tal vez, la mayor contribución de la dependencia.

Es en este contexto que reabrir el debate de la dependencia resulta significativo. Ahora bien, sería ingenuo pensar que el movimiento de la dependencia pudiese recobrar un reconocimiento como un marco analítico relevante en el estudio del desarrollo dentro de los discursos actuales, sin un importante trabajo de rectificación, refinación y reajuste. Dicho trabajo se enfrenta a incontables páginas de críticas de toda índole, y provenientes de diferentes campos, que representan diversos intereses. Muchas de estas críticas resultan superficiales, pero un grupo mayoritario presenta importantes observaciones a diferentes autores de la dependencia. Estas críticas invitan a pensar que el fallecimiento de este paradigma era necesario, y que sus aspectos relevantes, como muestra este artículo, siguieron vigentes en los estudios del desarrollo. En este sentido, podría parecer caprichoso e innecesario recuperar un debate que ya existe dentro de los discursos de desarrollo. Sin embargo, el hecho de que la dependencia haya influenciado muchísimos estudios actuales, o que muchos de estos estudios muestren importantes paralelismos con el movimiento, no reduce la necesidad de recuperar el paradigma como un marco analítico, precisamente porque permite contextualizar muchos de los trabajos modernos sobre desarrollo. Importantes aportes sobre la maldición de los recursos formulan y reformulan que la presencia de recursos naturales afecta negativamente al desarrollo. Pero fallan en discernir por qué algunos países con altos niveles de recursos primarios en sus actividades productivas, como Canadá, Noruega, Australia, o los Estados Unidos de América lograron trascender la situación condicionante de dependencia. Es más, estos trabajos también fallan en identificar las razones que imposibilitan, condicionan, o ralentizan acciones de cambio en países en situaciones de dependencia. Al contextualizar estos trabajos dentro del movimiento, se pueden responder muchas de estas preguntas, y mejorar el entendimiento sobre el desarrollo.

Por consiguiente, este artículo presenta distintas justificaciones y promueve una base teórica inicial para reabrir el debate de la dependencia. Las tendencias socioeconómicas actuales de América Latina presentan dinámicas similares a las que motivaron a los autores de la dependencia, y luego fueron explicadas por los mismos. La relación de China con la región, así como con otras como África, presenta características de dependencia que requieren de su estudio dentro de un marco teórico capaz de reconocer los factores condicionantes al desarrollo. Además, diferentes paradigmas sobre el estudio del mismo demuestran la validez, relevancia, e influencia de los postulados de la dependencia en la actualidad, que invitan a su discusión, 
reformulación, y aplicación ante las realidades empíricas modernas. Finalmente, la apertura del debate de la dependencia presenta importantes beneficios para paradigmas modernos establecidos como los estudios poscoloniales, brindándoles un contexto que en su momento decidieron omitir. Es evidente que el momento actual de la política económica latinoamericana requiere de un marco teórico capaz de problematizar y contextualizar las dislocaciones socioeconómicas que siguen caracterizando a la región, y la redefinición del movimiento de la dependencia puede dirigirse a estas necesidades.

\section{Referencias}

Acemoglu, D., Verdier, T., y Robinson, J. (2004). Kleptocracy and Divide-and-Rule: A Model of Personal Rule.Journal of The European Economic Association, 2(2-3), 162-192.

Bahar, D., y Santos, M. (2018). One More Resource Curse: Dutch Disease and Export Concentration. Journal of Development Economics, 132, 102-114. https://doi.org/10.1016/j.jdeveco.2018.01.002

Beigel, F. (2006). Vida, Muerte, y Resurrección de las “Teorías de la Dependencia”. En B. Levy (ed.), Crítica y Teoría en el Pensamiento Social Latinoamericano (pp. 287-326). Clacso.

Blaney, D. (1996). Reconceptualizing Autonomy: The Difference Dependency Theory Makes. Review of International Political Economy, 3(3), 459-497.

Blattman, C., Hwang, J., y Williamson, J. (2007). Winners and Losers in the Commodity Lottery: The Impact of Terms of Trade Growth and Volatility in the Periphery 1870-1939. Journal OfDevelopment Economics, 82(1), 156-179.

Boschini, A., Pettersson, J., y Roine, J. (2007). Resource Curse or Not: A Question of Appropriability. Scandinavian Journal of Economics, 109(3), 593-617.

Bourguignon, F., y Verdier, T. (2000). Oligarchy, Democracy, Inequality and Growth. Journal of Development Economics, 62(2), 285-313.

Bull, B., y Bøås, M. (2012). Between Ruptures and Continuity: Modernisation, Dependency and the Evolution of Development Theory. Forum for Development Studies, 39(3), 319-336.

Bulmer-Thomas, V. (1994). The Economic History of Latin America Since Independence. Cambridge University Press.

Burchardt, H., y Dietz, K. (2014). (Neo-)extractivism, a New Challenge for Development Theory from Latin America. Third World Quarterly, 35(3), 468-486.

Caporaso, J., y Zare, B. (1981). Interpretation and Evaluation of Dependency Theory. En H. Muñoz (ed.), From Dependency to Development (pp. 43-56). Westview Press.

Cardoso, F. (1973). Associated-dependent Development: Theoretical and Practical Implication. En A. Stepan (ed.), Authoritarian Brazil: Origins, Policies, and Future (pp. 142-176). Yale University Press.

Cardoso, F., y Faletto, E. (1979). Dependency and Development in Latin America. University of California Press.

Corden, M. (1984). Booming Sector and Dutch Disease Economics: Survey and Consolidation. Oxford Economic Papers, 36(3), 359-380.

Corden, W., y Neary, J. (1982). Booming Sector and De-Industrialisation in a Small Open Economy. The Economic Journal, 92(368), 825-848.

Deaton, A. (1999). Commodity Prices and Growth in Africa. Journal of Economic Perspectives, 13(3), 23-40.

Dos Santos, T. (1970). The Structure of Dependence. The American Economic Review, 60(2), 231-236.

Dos Santos, T. (1973). The Crisis of Development Theory and the Problem of Dependence in Latin America. En H. Bernstein (ed.), Underdevelopment and Development; The Third World Today. Penguin.

Duvall, R. (1978). Dependence and Dependency Theory. International Organization, 32(1), 51-78.

Evans, P. (1987). Class, State, and Dependence in East Asia: Lessons for Latin Americanists. In F. Deyo (ed.), The Political Economy of the New Asian Industrialism (pp. 203-226). Cornell University Press.

Frank, A. (1967). Capitalism and Underdevelopment in Latin America. Monthly Review Press. 
Furtado, C. (1973). La Economía Latinoamericana desde la Conquista Ibérica hasta la Revolución Cubana. Siglo XXI Editores.

Goderis, B., y Malone, S. (2011). Natural Resource Booms and Inequality: Theory and Evidence. Scandinavian Journal of Economics, 113(2), 388-417.

Godfrey, M. (1980). Editorial: Is Dependency Dead? The IDS Bulletin, 12(1), 1-4.

Gordon, W. (1965). The Political Economy of Latin America. Columbia University Press.

Hirschman, A. (1987). The Political Economy of Latin American Development: Seven Exercises in Retrospection. Latin American Research Review, 22(3), 7-36.

Isham, J., Woolcock, M., Pritchett, L., y Busby, G. (2005). The Varieties of Resource Experience: Natural Resource Export Structures and the Political Economy of Economic Growth. World Bank Economic Review, 19(2), 141-174.

Jaguaribe, H., Ferrer, A., y Wionczek, M. (1968) La dependencia poli\#tico-econo\#mica de Ame\#rica Latina. Siglo XXI Editores.

Kay, C. (1989). Latin American Theories of Development and Underdevelopment. Routledge.

Muñoz, H. (1981). From Dependency to Development. Westview Press.

OECD, Eclac, y CAF. (2016). Latin American Economic Outlook 2017: Youth, Skills and Entrepreneurship. OECD Publishing.

Ortiz, J. (2012). Déjà vu: Latin America and Its New Trade Dependency...This Time with China. Latin American Research Review, 47(3), 175-190.

Packenham, R. (1992). The Dependency Movement. Harvard University Press.

Palma, G. (1978). Dependency: a formal theory of underdevelopment or a methodology for the analysis of concrete situations of underdevelopment? World Development, 6(7-8), 881-924.

Programa de las Naciones Unidas para el Desarrollo. (2016). Reporte del desarrollo humano de América Latina y el Caribe. http://hdr.undp.org/en/content/human-development-report-latin-america-and-carribbean-2016

Sachs, J., y Warner, A. (1995). Natural Resource Abundance and Economic Growth. En G. Meier y J. Rauch (eds.), Leading Issues in Economic Development (pp. 161-167). Oxford University Press.

Sánchez, O. (2003). The Rise and Fall of the Dependency Movement. Estudios Interdisciplinarios de América Latina, 14(2), 31-50.

Skidmore, T., y Smith, P. (1992) Modern Latin America. Oxford University Press.

Smith, P. (2012). Democracy in Latin America: Political Change in Comparative Perspective. Oxford University Press.

Tavares, R. (2017). Los Latinoamericanos están volviendo a caer en la pobreza, pero las inversiones de impacto pueden revertir esa preocupante tendencia. World Economic Forum on Latin America. https://www.weforum.org/es/agenda/2017/04/los-latinoamericanos-estan-volviendo-a-caer-en-la-p obreza-pero-las-inversiones-de-impacto-pueden-revertir-esa-preocupante-tendencia/

Tsounta, E., y Osueke, A. (2014). What Is Behind Latin America's Declining Income Inequality? (Documento de trabajo WP/14/124). Fondo Monetario Internacional.

Van der Ploeg, F., y Poelhekke, S. (2009). Volatility and the Natural Resource Curse. Oxford Economic Papers, 61(4), 727-760.

\section{Notas}

* Artículo de investigación

\section{Licencia Creative Commons CC BY 4.0}

Cómo citar este artículo: Zambrano, D. (2020). La influencia de la teoría de la dependencia en los discursos de desarrollo de América Latina. Papel Político, 25. https://doi.org/10.11144/Javeriana.papo25.itdd 